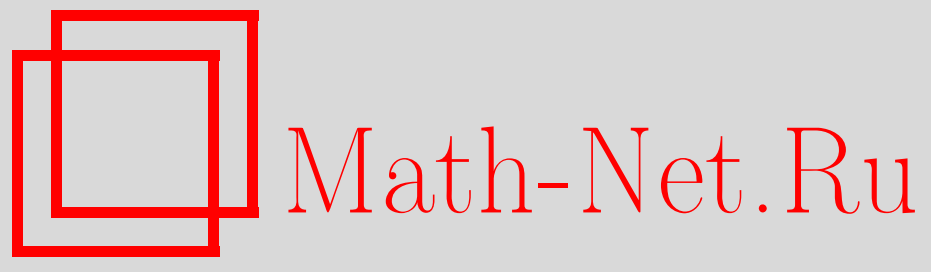

В. В. Журавлёва, О двух наименьших числах Пизо, $M a-$ тем. заметки, 2013, том 94, выпуск 5, 784-787

DOI: https://doi.org/10.4213/mzm10336

Использование Общероссийского математического портала Math-Net.Ru подразумевает, что вы прочитали и согласны с пользовательским соглашением http://www . mathnet.ru/rus/agreement

Параметры загрузки:

IP: 3.91 .87 .62

26 апреля 2023 г., 15:49:03

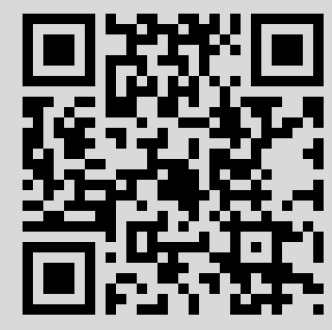




\section{О двух наименьших числах Пизо}

\section{В. В. Журавлева}

1. Введение. Напомним, что числом Пизо называется целое алгебраическое число, такое что оно само больше 1 , а его сопряженные лежат строго внутри единичного круга $\mathbb{D}=\{z \in \mathbb{C}:|z|<1\}$.

Числа Пизо могут быть охарактеризованы с помощью их диофантовых свойств. Известен следующий результат [1; гл. VIII].

Теорема 1. 1) Если $\theta$ - число Пизо, а $\lambda$ - иелое алгебраическое из поля $\mathbb{Q}(\theta)$, то последовательность $\left\|\lambda \theta^{n}\right\| \rightarrow 0$ при $n \rightarrow \infty$.

2) Если $\theta>1$ - алгебраическое число, $\lambda \neq 0$ - действительное и последовательность $\left\|\lambda \theta^{n}\right\| \rightarrow 0$ при $n \rightarrow \infty$, то $\theta$ - число Пизо, $а \lambda \in \mathbb{Q}(\theta)$.

(Здесь и далее $\|\cdot\|$ обозначает расстояние до ближайшего целого, в то время как $\{\cdot\}$ это дробная часть.)

Диофантовым свойствам чисел Пизо посвящено достаточно много работ (см. [2]-[4]). В частности, в своей статье автор [5], рассматривая величину

$$
L(\theta):=\sup _{\xi \in \mathbb{R}} \liminf _{n \rightarrow \infty}\left\|\xi \theta^{n}\right\|
$$

в случае $\theta=(\sqrt{5}+1) / 2$, доказал следующее утверждение.

Теорема 2. Выполнено равенство $L((\sqrt{5}+1) / 2)=1 / 5$.

Настоящая статья посвящена вычислению величины $L(\theta)$ для максимального корня уравнения $x^{3}-x-1=0$. Известно (см. работу К. Зигеля [6]), что это число является самым маленьким числом Пизо.

Теорема 3. Пусть $\theta$ - наибольший корень уравнения $x^{3}-x-1=0$, тогда выполнено $L(\theta)=1 / 5$.

Для доказательства этой теоремы достаточно сделать следующее:

1) установить существование такого $\xi$, что $\liminf _{n \rightarrow \infty}\left\|\xi \theta^{n}\right\|=1 / 5$;

2 ) построить функцию $U(x, y, z)$ такую, что $L(\theta) \leqslant \max _{x, y, z \in \mathbb{R}} U(x, y, z)=1 / 5$; а именно, такой функцией является

$$
\begin{aligned}
U(x, y, z)=\min _{x, y, z \in \mathbb{R}}\{\|x\|,\|y\|,\|z\|,\|x+y\|,\|y+z\|, \\
\qquad x+y+z\|,\| x+2 y+z\|,\| x+2 y+2 z\|,\| 2 x+3 y+2 z \|\} .
\end{aligned}
$$

Исходя из этого, данная статья организована следующим образом. Во втором пункте мы докажем первое утверждение. Затем перейдем к доказательству второго утверждения. Следует отметить, что в его доказательстве мы опираемся на результаты компьютерных вычислений.

Аналогичным образом может быть получен результат для второго по минимальности числа Пизо (см. [6]).

Теорема 4. Пусть $\theta$ - наибольший корень уравнения $x^{4}-x^{3}-1=0$. Тогда выполнено $L(\theta)=3 / 17$.

Работа выполнена при поддержке Российского фонда фундаментальных исследований (гранты № 12-01-00681-a).

DOI: $10.4213 / \mathrm{mzm} 10336$ 
2. Оценка снизу. Пусть $\theta, \theta_{2}, \theta_{3}-$ корни уравнения $x^{3}-x-1=0$. Заметим, что последовательности

$$
\begin{array}{lll}
A=\left(a_{n}\right)_{n=1}^{\infty}, & \text { где } & a_{n}=\theta_{2}^{n-1}, \\
B=\left(b_{n}\right)_{n=1}^{\infty}, & \text { где } & b_{n}=\theta_{3}^{n-1}, \\
D=\left(d_{n}\right)_{n=1}^{\infty}, & \text { где } & d_{n}=\xi \theta^{n-1}
\end{array}
$$

( $\xi$ - любое действительное число) удовлетворяют рекуррентному соотношению

$$
x_{n}=x_{n-2}+x_{n-3}, \quad n \geqslant 4 .
$$

Очевидно, что $\lim _{n \rightarrow \infty} a_{n}=\lim _{n \rightarrow \infty} b_{n}=0$. Также любая последовательность, являющаяся линейной комбинацией $A$ и $B$, будет рекуррентной с характеристическим уравнением $x^{3}-x-1=0$ и стремится к 0 . Каждая последовательность, удовлетворяющая рекуррентному соотношению $x_{n}=x_{n-2}+x_{n-3}$ при $n \geqslant 4$, однозначно задается тремя своими первыми членами.

Точки $(0,0,0),\left(1, \theta_{2}, \theta_{2}^{2}\right),\left(1, \theta_{3}, \theta_{3}^{2}\right)$ лежат в плоскости $x+\theta^{2} y+\theta z=0 . \quad$ Поэтому, если $(x, y, z)$ принадлежит этой плоскости, то для рекуррентной последовательности $X=$ $\left(x_{n}\right)_{n=1}^{\infty}$ с характеристическим уравнением $x^{3}-x-1=0$ и $x_{1}=x, x_{2}=y, x_{3}=z$ выполнено $\lim _{n \rightarrow \infty}\left\|x_{n}\right\|=0$.

Легко видно, что для последовательности $C=\left(c_{n}\right)_{n=1}^{\infty}$, удовлетворяющей рекуррентному соотношению

$$
c_{n}=c_{n-2}+c_{n-3}, \quad n \geqslant 4,
$$

с $c_{1}=2 / 5, c_{2}=4 / 5, c_{3}=3 / 5$ выполняются равенства

$$
\left\{c_{4 k-3}\right\}=\frac{2}{5}, \quad\left\{c_{4 k-2}\right\}=\frac{4}{5}, \quad\left\{c_{4 k-1}\right\}=\frac{3}{5}, \quad\left\{c_{4 k}\right\}=\frac{1}{5}, \quad k \in \mathbb{N} .
$$

Следовательно, $\liminf _{n \rightarrow \infty}\left\|c_{n}\right\|=1 / 5$.

Из вышесказанного следует, что если

$$
\left(d_{1}-\frac{2}{5}\right)+\theta^{2}\left(d_{2}-\frac{4}{5}\right)+\theta\left(d_{3}-\frac{3}{5}\right)=0
$$

то

$$
\lim _{n \rightarrow \infty}\left(\left\{c_{n}\right\}-\left\{d_{n}\right\}\right)=0
$$

Таким образом, если взять

$$
\xi=\frac{2+4 \theta^{2}+3 \theta}{5\left(1+2 \theta^{3}\right)}
$$

то $\lim \inf _{n \rightarrow \infty}\left\|d_{n}\right\|=1 / 5$. Тем самым, доказана оценка снизу $L(\theta) \geqslant 1 / 5$.

3. Оценка сверху. Для доказательства второго утверждения мы будем исследовать график функции

$$
\begin{aligned}
F(x, y, z):=\min \{\|x\|,\|y\|,\|z\|,\|x+y\|,\|y+z\|, \\
\|x+y+z\|,\|x+2 y+z\|,\|x+2 y+2 z\|,\|2 x+3 y+2 z\|\}
\end{aligned}
$$

при $0 \leqslant x, y, z<1$. Он состоит из кусков трехмерных аффинных плоскостей. Очевидно, что экстремумы $F(x, y, z)$ могут находиться только в точках пересечения 4 таких плоскостей.

Основное УтвеРЖДЕнИЕ. Максимум функции $F(x, y, z)$ достигается в 4 следующих mочках:

$$
\begin{aligned}
\left(x_{1}, y_{1}, z_{1}\right):=\left(\frac{1}{5}, \frac{2}{5}, \frac{4}{5}\right), & \left(x_{2}, y_{2}, z_{2}\right):=\left(\frac{2}{5}, \frac{4}{5}, \frac{3}{5}\right) \\
\left(x_{3}, y_{3}, z_{3}\right):=\left(\frac{4}{5}, \frac{3}{5}, \frac{1}{5}\right), & \left(x_{4}, y_{4}, z_{4}\right):=\left(\frac{3}{5}, \frac{1}{5}, \frac{2}{5}\right) .
\end{aligned}
$$


Основное утверждение состоит из нескольких вспомогательных утверждений.

Утверждение 5. Для доказательства $F(x, y, z) \leqslant 1 / 5$ nри $0 \leqslant x, y, z<1$ достаточно доказать $F(x, y, z) \leqslant 1 / 5$ при $1 / 5 \leqslant x, y, z \leqslant 4 / 5$.

Это следует из очевидного наблюдения, что при $\min \{\|x\|,\|y\|,\|z\|\}<1 / 5$ значение функции можно оценить сверху: $F(x, y, z) \leqslant \min \{\|x\|,\|y\|,\|z\|\}<1 / 5$.

Результаты компьютерных вычислений. Пройдя область $1 / 5 \leqslant x, y, z \leqslant 4 / 5$ с шагом 1/1000 по всем трем направлениям, было получено неравенство

$$
F(x, y, z)<\frac{1}{5}-\frac{4}{1000}, \quad \text { если } \quad(x, y, z) \notin \mathcal{F}_{i}, \quad \text { где } \quad i=1,2,3,4,
$$

и

$$
\begin{aligned}
& \mathcal{F}_{1}=\left\{\begin{array}{l}
\left|x-\frac{1}{5}\right| \leqslant \frac{12}{1000}, \\
\left|y-\frac{2}{5}\right| \leqslant \frac{12}{1000}, \\
\left|z-\frac{4}{5}\right| \leqslant \frac{12}{1000} ;
\end{array} \quad \mathcal{F}_{2}=\left\{\begin{array}{l}
\left|x-\frac{2}{5}\right| \leqslant \frac{12}{1000}, \\
\left|y-\frac{4}{5}\right| \leqslant \frac{12}{1000}, \\
\left|z-\frac{3}{5}\right| \leqslant \frac{12}{1000} ;
\end{array}\right.\right. \\
& \mathcal{F}_{3}=\left\{\begin{array}{l}
\left|x-\frac{4}{5}\right| \leqslant \frac{12}{1000}, \\
\left|y-\frac{3}{5}\right| \leqslant \frac{12}{1000}, \\
\left|z-\frac{1}{5}\right| \leqslant \frac{12}{1000} ;
\end{array} \quad \mathcal{F}_{4}=\left\{\begin{array}{l}
\left|x-\frac{3}{5}\right| \leqslant \frac{12}{1000}, \\
\left|y-\frac{1}{5}\right| \leqslant \frac{12}{1000}, \\
\left|z-\frac{2}{5}\right| \leqslant \frac{12}{1000} .
\end{array}\right.\right.
\end{aligned}
$$

УтвеРЖДЕниЕ 6. Если в вершинах куба с длинами сторон, равными 1/1000, значения $F(x, y, z)<1 / 5-4 / 1000$, то и во всех точках внутри этого куба $F(x, y, z)<1 / 5$.

Заметим, что $\partial F / \partial x+\partial F / \partial y+\partial F / \partial z \leqslant 7$. Самая удаленная точка от вершин куба находится на расстоянии 5/10000 от каждой из вершин. В ней

$$
F(x, y, z)<\frac{1}{5}-\frac{4}{1000}+7 \frac{5}{10000}<\frac{1}{5} .
$$

Осталось рассмотреть области $\mathscr{F}_{i}, i=1,2,3,4$. В них достаточно точно построить график функции. Подробно опишем область $\mathscr{F}_{1}(x, y, z)$. Для остальных рассуждения аналогичны.

Анализ области $\mathscr{F}_{1}$. В области $\mathscr{F}_{1}(x, y, z)$ выполнено

$$
\begin{array}{rlrl}
u_{1}(x, y, z):=\|x\|=x, & & u_{4}(x, y, z):=\|x+y\|=1-x-y, \\
u_{2}(x, y, z):=\|y\|=y, & & u_{5}(x, y, z):=\|y+z\|=y+z-1, \\
u_{3}(x, y, z):=\|z\|=1-z, & & u_{6}(x, y, z):=\|x+y+z\|=x+y+z-1, \\
& u_{7}(x, y, z):=\|x+2 y+z\|=2-x-2 y-z, \\
& u_{8}(x, y, z):=\|x+2 y+2 z\|=3-x-2 y-2 z \\
& u_{9}(x, y, z):=\|2 x+3 y+2 z\|=2 x+3 y+2 z-3 .
\end{array}
$$

Функции $u_{i}(1 / 5,2 / 5,4 / 5)=1 / 5$ при нечетных $i$ и $2 / 5$ при четных $i$. Следовательно, в формировании максимума участвуют только линейные формы с нечетными индексами.

Область $\mathscr{F}_{1}$ - это куб. Разделим его на 5 областей в зависимости от того, какая из функций $u_{i}(x, y, z), i=1,3,5,7,9$, минимальна. Таким построением мы и определим точку, в которой достигается максимум. Ею будет $(1 / 5,2 / 5,4 / 5)$. Отметим, что в этой точке пересекаются все 5 областей. 


\section{СПИСОК ЦИТИРОВАННОЙ ЛИТЕРАТУРЫ}

[1] J.W.S. Cassels, An Introduction to Diophatine Approximations, Cambridge Tracts in Math. and Math. Phys., 45, Cambridge Univ. Press, Cambridge, 1957. [2] T. Vijayaraghavan, Proc. Cambridge Phil. Soc., 37 (1941), 349-357. [3] A. Dubickas, Bull. London Math. Soc., 38:1 (2006), 70-80. [4] M. J. Bertin, A. Decomps-Guilloux, M. Grandet-Hugot, M. Pathiaux-Delefosse, J.P. Schreiber, Pisot and Salem Numbers, Birkhäuser Verlag, Basel, 1992. [5] V. Zhuravleva, J. Théor. Nombres Bordeaux, 25 (2013), 499-520. [6] C. L. Siegel, Duke Math. J., 11:3 (1944), 597-602.

E-mail: victoria.zhuravleva@me.com 